\title{
Engineering biphoton wave packets with an electromagnetically induced grating
}

\author{
Jianming Wen, ${ }^{1,2, *}$ Yan-Hua Zhai, ${ }^{3}$ Shengwang Du, ${ }^{4}$ and Min Xiao ${ }^{1,2}$ \\ ${ }^{1}$ Department of Physics, National Laboratory of Solid State Microstructures, Nanjing University, Nanjing 210093, China \\ ${ }^{2}$ Department of Physics, University of Arkansas, Fayetteville, Arkansas 72701, USA \\ ${ }^{3}$ Department of Physics, University of Maryland, Baltimore County, Baltimore, Maryland 21250, USA \\ ${ }^{4}$ Department of Physics, The Hong Kong University of Science and Technology, Clear Water Bay, Kowloon, Hong Kong, China
}

(Received 20 July 2010; published 12 October 2010)

\begin{abstract}
We propose to shape biphoton wave packets with an electromagnetically induced grating in a four-level double- $\Lambda$ cold atomic system. We show that the induced hybrid grating plays an essential role in directing the new fields into different angular positions, especially for the zeroth-order diffraction. A number of interesting features appears in the shaped two-photon wave forms. For example, broadening or narrowing the spectrum would be possible in the proposed scheme even without the use of a cavity.
\end{abstract}

DOI: 10.1103/PhysRevA.82.043814 PACS number(s): 42.50.Dv, 42.65.Lm, 42.50.Ct, 03.65.Ud

\section{INTRODUCTION}

The generation of entangled paired photons with a desired joint spectrum has become a fascinating conceptual viewpoint for both fundamental and practical research. This is because the joint spectrum contains the information on bandwidth, type of frequency correlations, and wave function of the two-photon state. By manipulating the joint spectrum, one can obtain the most appropriate form for the specific quantum optics application under consideration. For instance, biphotons with a narrow bandwidth play a key role in the long-distance quantum communication protocols based on an atom-photon interface [1]; biphotons with a few femtoseconds of correlation time are of particular interest in the fields of quantum metrology [2] and for some protocols for timing and positioning measurements [3].

Conventionally, entangled paired photons are produced from the process of spontaneous parametric down-conversion (SPDC) in a nonlinear crystal, where a pump photon is annihilated and two down-converted daughter photons are simultaneously emitted [4]. Because of their broad bandwidth and short coherence time, it is difficult to shape SPDC photon wave packets in the time domain directly. A number of methods has been proposed and developed to perform spectral manipulation of the joint spectrum [5-7] or spatial modulation of the nonlinear interaction [8-10]. Others are to modify the (quasi-)phase matching [11], engineer the dispersive properties of the nonlinear medium [12], or imprint the spectral and spatial characteristics of the pump beam into the joint spectrum [13].

A recent demonstration of the generation of narrow-band biphotons in cold atomic ensembles via spontaneous four-wave mixing (SFWM) [14-18] has attracted considerable attention because of their long coherence time and controllable quantum wave packets. Nonlocal modulation of temporal correlation has been observed with such narrow-band biphotons [19]. In a very recent experiment [20], shaping of the temporal wave form by periodically modulating the input driving lasers has confirmed the previous theoretical prediction [21], in which the input field profiles can be revealed in the

*jianming.wen@gmail.com two-photon correlation measurements. One major advantage over shaping the SPDC photon temporal wave function is that these narrow-band biphotons allow further wave-packet modification directly in the time domain.

In this paper, we describe a way to manipulate paired Stokes and anti-Stokes wave forms produced from SFWM in a four-level double- $\Lambda$ [22] cold atomic system with the use of an electromagnetically induced grating (EIG) [23,24]. EIG has been experimentally demonstrated in cold atoms [25,26] and has been applied to all optical switching and routing in hot atomic vapors [27]. Here, we show that, by spatially modulating the control beams, alternating regions of high transmission and absorption can be created inside the atomic sample that act as an amplitude grating and by which the joint Stokes and anti-Stokes wave packet can be shaped. Compared with previous proposals ascribed before, several interesting features appear in the present one. First, such a medium may exert both amplitude and phase modulations on biphoton wave packets in much the same way that a hybrid (amplitude and phase) grating does to the amplitude and phase of an electromagnetic wave. Second, the spatial modulation of the control fields is imprinted into both the linear and the nonlinear susceptibilities. Consequently, this mapping may broaden or may narrow the joint spectrum depending on the system's parameters. Third, but not least, because of the grating diffraction interference, the spectral brightness can be improved, and the emission angle can be confined to some particular angles. For example, the anti-Stokes field will be mainly directed to the zeroth-order diffraction.

We organize the paper as follows. The basic idea is presented in Sec. II by considering two-photon temporal correlation measurement. The conclusion is summarized in Sec. III.

\section{SHAPING BIPHOTON WAVE FORM WITH EIG}

\section{A. EIG}

To illustrate the basic idea, we consider a four-level double$\Lambda$ atomic system (e.g., ${ }^{87} \mathrm{Rb}$ ) depicted in Fig. 1(a), where all the atomic population is assumed to be in the ground state $|1\rangle$. To ignore the Doppler broadening, the atoms are laser cooled in a magnetic optical trap. Two strong control 


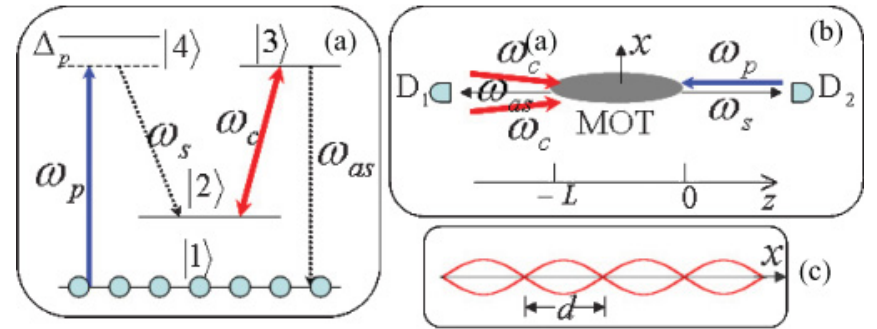

FIG. 1. (Color online) Shaping biphoton wave packets with an EIG. (a) The level structure, where in the presence of a $\mathrm{cw}$ probe $\left(\omega_{p}\right)$ and control $\left(\omega_{c}\right)$ fields, paired Stokes $\left(\omega_{s}\right)$ and anti-Stokes $\left(\omega_{a s}\right)$ photons are spontaneously created from the four-wave mixing processes in the low-gain regime. (b) The backward generation geometry, where two strong control beams symmetrically displace with respect to $z$ and form a standing wave along $x$. (c) The standing wave formed by control fields.

fields $\left(\omega_{c}\right)$, resonant with the atomic transition $|2\rangle \rightarrow|3\rangle$ while being symmetrically displaced with respect to $z$, are incident upon the atomic ensemble at such angles that they intersect and form a standing wave within the medium [see Fig. 1(c)]. In the presence of the counterpropagating weak probe field $\left(\omega_{p}\right)$ far detuned from the transition $|1\rangle \rightarrow|4\rangle$, phase-matched Stokes $\left(\omega_{s}\right)$ and anti-Stokes $\left(\omega_{a s}\right)$ photons are then spontaneously generated in opposite directions and are detected by single-photon detectors $\mathrm{D}_{2}$ and $\mathrm{D}_{1}$, respectively, as shown in Fig. 1(b). Since the linear and nonlinear optical responses to the generated fields depend on the strength of the control light, they are expected to change periodically as the standing wave changes from the nodes to the antinodes across the $x$ dimension. In the current configuration, the Stokes photons travel at nearly the speed of light in vacuum with negligible Raman gain. In contrast, the strong control beams induce a set of periodic transparency windows to the anti-Stokes field. Thus, alternatively, a nonmaterial grating is formed in the anti-Stokes channel. This grating is termed as an EIG [23], which will diffract the anti-Stokes field into some particular angles according to the diffraction orders.

Following the analysis presented in Ref. [17], the thirdorder nonlinear susceptibility for the generated anti-Stokes field is calculated to be

$$
\chi_{a s}^{(3)}(\omega)=\frac{-N \mu_{13} \mu_{32} \mu_{24} \mu_{41} /\left[4 \hbar^{3} \epsilon_{0}\left(\Delta_{p}+i \gamma_{41}\right)\right]}{\left(\omega-\Omega_{e}+i \gamma_{e}\right)\left(\omega+\Omega_{e}+i \gamma_{e}\right)},
$$

and the linear susceptibilities at the Stokes and anti-Stokes frequencies are, respectively,

$$
\begin{gathered}
\chi_{s}(\omega)=\frac{N\left|\mu_{42}\right|^{2}\left(\omega-i \gamma_{31}\right) /\left(4 \hbar \epsilon_{0}\right)}{\left|\Omega_{c}\right|^{2} \cos ^{2}\left(\frac{\pi x}{d}\right)-\left(\omega-i \gamma_{31}\right)\left(\omega-i \gamma_{21}\right)} \frac{\left|\Omega_{p}\right|^{2}}{\Delta_{p}^{2}+\gamma_{41}^{2}}, \\
\chi_{a s}(\omega)=\frac{N\left|\mu_{31}\right|^{2}\left(\omega+i \gamma_{21}\right) /\left(\hbar \epsilon_{0}\right)}{\left|\Omega_{c}\right|^{2} \cos ^{2}\left(\frac{\pi x}{d}\right)-\left(\omega+i \gamma_{31}\right)\left(\omega+i \gamma_{21}\right)},
\end{gathered}
$$

where $N$ is the atomic density, $\mu_{i j}$ are the dipole matrix elements, $\Omega_{p}$ and $\Omega_{c}$ are the probe and control Rabi frequencies, $\gamma_{i j}$ are the decay or dephasing rate, $\Delta_{p}=\omega_{p}-\omega_{41}$ is the probe detuning, and $d=\frac{\pi}{k_{c x}}$ represents the space period, which can be made arbitrarily larger than the wavelength of the control fields by varying the angle between their two wave

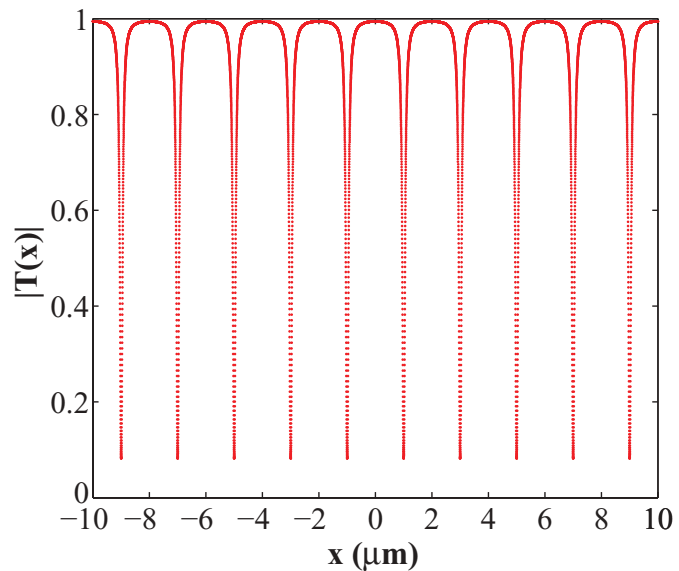

FIG. 2. (Color online) A typical transmission profile of the antiStokes field as a function of $x$. Parameters are chosen as $d=2 \mu \mathrm{m}$, the optical depth about $5, \gamma_{31}=2 \pi \times 3 \mathrm{MHz}$, and $\gamma_{21}=0.6 \gamma_{31}$.

vectors. $\Omega_{e}=\sqrt{\left|\Omega_{c}\right|^{2} \cos ^{2}\left(\frac{\pi x}{d}\right)+\gamma_{31} \gamma_{21}} \approx\left|\Omega_{c}\right| \cos \left(\frac{\pi x}{d}\right)$ is the effective control Rabi frequency, and $\gamma_{e}=\frac{\gamma_{31}+\gamma_{21}}{2}$ is the effective dephasing rate. $\chi_{a s}^{(3)}$ in Eq. (1) has two resonances separated by $\Omega_{e}$, and each is associated with a linewidth of $2 \gamma_{e}$. From Eqs. (1) and (2), it is obvious that the spatial periodic modulation of the control fields has been mapped into the optical responses to the Stokes and antiStokes fields. Consequently, such a modulation will further modify the two-photon wave form as will be discussed later. It is known that the linear susceptibilities determine the transmission bandwidth and dispersion property. Taking $\left|\Omega_{p}\right| \ll \Delta_{p}, \chi_{s}$ is approximated as 0 , which means the Stokes photons traverse the medium almost at the speed of light in vacuum, and the Raman gain is negligible. In contrast, the anti-Stokes photons may propagate at a lower group velocity $v_{g} \approx 2 \hbar \epsilon_{0} c\left|\Omega_{c}\right|^{2} \cos ^{2}\left(\frac{\pi x}{d}\right) / N\left|\mu_{31}\right|^{2} \omega_{31}=v_{0} \cos ^{2}\left(\frac{\pi x}{d}\right)$ and may experience periodic linear loss characterized by $\alpha=N \sigma_{31} \gamma_{21} \gamma_{31} / 2\left[\left|\Omega_{c}\right|^{2} \cos ^{2}\left(\frac{\pi x}{d}\right)+\gamma_{21} \gamma_{31}\right]$, where $\sigma_{31}=$ $\omega_{31}\left|\mu_{31}\right|^{2} /\left(\hbar \epsilon_{0} c \gamma_{31}\right)$ is the on-resonance absorption cross section in the transition $|1\rangle \rightarrow|3\rangle$.

Thus, such a periodic linear loss results in an EIG to the anti-Stokes photons. Figure 2 displays a typical transmission function for the anti-Stokes light as a function of $x$. It is easy to understand that, at the transverse locations around the nodes (of the standing wave), the control field intensities are so weak that the anti-Stokes field is absorbed according to the usual Beer law. In contrast, since the intensity distribution of the control fields at the spatial locations around the antinodes is very strong, the absorption of the anti-Stokes field is greatly suppressed due to the effect of electromagnetically induced transparency [28]. This leads to a periodic amplitude modulation across the beam profile of the anti-Stokes light, a phenomenon reminiscent of the amplitude grating.

\section{B. Shaping two-photon wave form}

The paired Stokes and anti-Stokes photon states can be obtained from first-order perturbation theory [16,17,29]. For simplicity, we take the input probe and control beams as classical cw lasers and focus on the two-photon temporal 
correlation. The effective interaction length is taken as $L$. The unnormalized biphoton state at the output surfaces of the sample may be written as

$$
|\Psi\rangle=\Psi_{0} \int d \omega \Phi(\omega) a_{s}^{\dagger} a_{a s}^{\dagger}|0\rangle,
$$

where $\Psi_{0}$ is a grouped constant, and the joint spectral function takes the form

$$
\Phi(\omega)=\int_{x_{1}}^{x_{2}} d x \chi_{a s}^{(3)}(\omega) \cos \left(\frac{\pi x}{d}\right) \operatorname{sinc}\left(\frac{\Delta k L}{2}\right) e^{i(\Delta k L / 2)},
$$

where the cosine term comes from the standing wave of the control fields, and the last two terms come from the longitudinal phase-matching condition with $\Delta k \approx \frac{\omega}{v_{g}}+i \alpha$. In Eq. (4), we have taken the linear loss into account. It is clear that the joint spectrum $\Phi$ can be engineered through $\chi_{a s}^{(3)}$ and the phase-matching condition. After, we describe the wave-packet shaping by considering the simple two-photon temporal correlation measurement in which paired Stokes and anti-Stokes photons are detected by single-photon detectors $\mathrm{D}_{1}$ and $\mathrm{D}_{2}$ with equal pathways from the output surfaces of the medium, as shown in Fig. 1(b). Since there are two characteristic timings embedded in Eq. (4), the resonance linewidth determined by $\chi_{a s}^{(3)}$ and the natural spectral width determined by the phase-matching condition $\frac{L}{v_{0}}$ will be looked at separately by using the two-photon temporal correlation in which only one characteristic timing is dominant.

Using the Glauber theory, the two-photon amplitude is

$$
A=\left\langle 0\left|E_{s}^{(+)} E_{a s}^{(+)}\right| \Psi\right\rangle .
$$

The field $E_{j}^{(+)}$is the positive-frequency part of the free-space electromagnetic field at position $r_{j}$ and time $t_{j}$. In the far-field region (Fraunhofer diffraction), the biphoton amplitude (5) over the diffraction angle $\theta$ (with respect to $z$ ) can be derived, by following the procedure done in Refs. [17,18,30], as

$$
\begin{aligned}
A(\tau ; \theta)= & A \int_{x_{1}}^{x_{2}} d x \cos \left(\frac{\pi x}{d}\right) e^{i k_{a s} x \sin \theta} \\
& \times \int d \omega \chi_{a s}^{(3)}(\omega) \operatorname{sinc}\left(\frac{\Delta k L}{2}\right) e^{i(\Delta k L / 2-\omega \tau)},
\end{aligned}
$$

where $A$ is an integrant-irrelevant constant, $\tau=t_{a s}-t_{s}$ is the relative time delay between two clicks, and $k_{a s}$ is the central wave number of the anti-Stokes photons. Equation (6) can further be recast into a product of an integral and a geometric series,

$$
\begin{aligned}
A(\tau ; \theta)= & A \sum_{n=-M / 2}^{M / 2} e^{i k_{a s} n d \sin \theta} \int_{-d / 2}^{d / 2} d x \cos \left(\frac{\pi x}{d}\right) e^{i k_{a s} x \sin \theta} \\
& \times \int d \omega \chi_{a s}^{(3)}(\omega) \operatorname{sinc}\left(\frac{\Delta k L}{2}\right) e^{i(\Delta k L / 2-\omega \tau)},
\end{aligned}
$$

where $M$ represents the input probe field across $M$ times $d$. This can be guaranteed by adjusting the diameters of both probe and control fields to cover $M$ slits. By evaluating the geometric progression in the usual fashion, Eq. (7) can be written as

$$
A(\tau ; \theta)=A \frac{\sin \frac{k_{a s} M d \sin \theta}{2}}{\sin \frac{k_{a s} d \sin \theta}{2}} B(\tau ; \theta),
$$

with

$$
\begin{aligned}
B(\tau ; \theta)= & \int_{-d / 2}^{d / 2} d x \cos \left(\frac{\pi x}{d}\right) e^{i k_{a s} x \sin \theta} \\
& \times \int d \omega \chi_{a s}^{(3)}(\omega) \operatorname{sinc}\left(\frac{\Delta k L}{2}\right) e^{i(\Delta k L / 2-\omega \tau)} .
\end{aligned}
$$

Therefore, the diffracted two-photon amplitude is a product of a single slit Eq. (9) multiplied by the function in Eq. (8). Equations (8) and (9) together imply that the two correlated Stokes and anti-Stokes photons are simultaneously produced from any one of the slits, which can be regarded as a superposition of coherent SFWM subsources. We also notice that the first integration in Eq. (9) can be visualized as an amplitude grating with a transmission profile followed by a cosine curvature. The emission angles and diffraction efficiencies are determined by the ability of the induced grating. From Eq. (8), it is easy to obtain the diffraction angles of the anti-Stokes field for different diffraction orders $m$ as

$$
\sin \theta=m \frac{\lambda_{a s}}{d},
$$

where $\lambda_{a s}=2 \pi / k_{a s}$. According to the results shown in Ref. [23], the diffraction mainly occurs at the zeroth order. This could be important to direct the light into a smaller solid angle and, hence, enhance its spectral brightness at the observation's location. Equations (8) and (9) are our starting points to analyze shaping of biphoton wave forms using EIG. Since, for the anti-Stokes field, the energy is almost emitted toward the zeroth-order diffraction direction, we assume $\theta=0$ in Eq. (8) to simplify the analysis in the following.

\section{Two-photon coincidence counts}

First, let us look at the case in which the coherence time is mainly determined by the resonance linewidth. In such a case, the natural spectral width from the phase matching is much greater than the linewidth. Hence, its effect on two-photon temporal correlation can be ignored. Thus, Eq. (9) reduces to

$$
B(\tau)=\int_{-d / 2}^{d / 2} d x \cos \left(\frac{\pi x}{d}\right) \int d \omega \chi_{a s}^{(3)}(\omega) e^{-i \omega \tau} .
$$

Plugging Eq. (1) into Eq. (11) and completing the frequency integral yields

$$
B(\tau)=B \int_{-d / 2}^{d / 2} d x \sin \left[\left|\Omega_{c}\right| \tau \cos \left(\frac{\pi x}{d}\right)\right] e^{-\gamma_{e} \tau},
$$

where $B$ is a constant. Different from previous findings [14-18,20,21,30], Eq. (12) clearly shows that the profile of the biphoton wave form is further manipulated by the periodic modulation of the control fields. Implementing the integration in Eq. (12) gives

$$
B(\tau)=B d H_{0}\left(\left|\Omega_{c}\right| \tau\right) e^{-\gamma_{e} \tau},
$$

where $H_{0}(x)$ is the Struve function of order zero. The two-photon coincidence counting rate equals the square of 


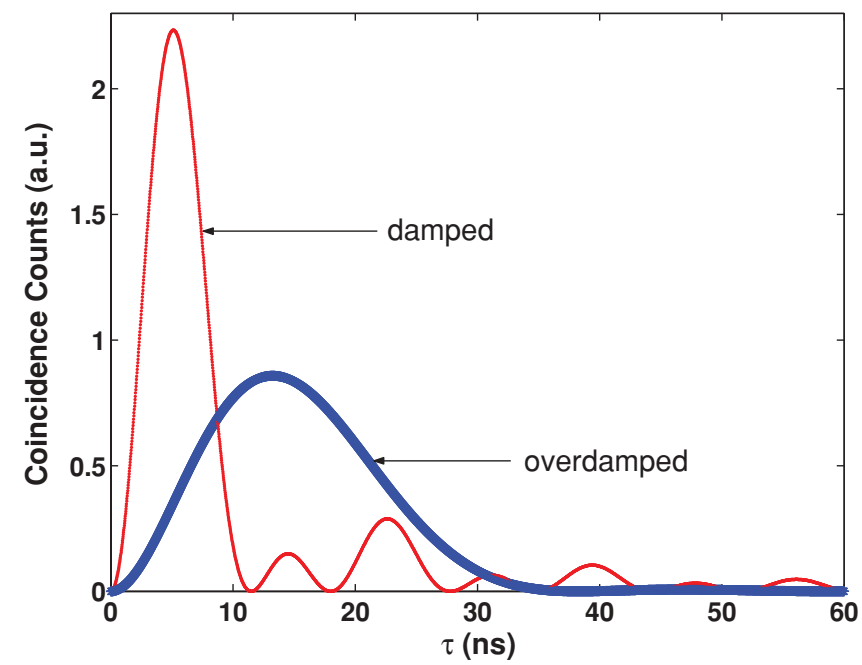

FIG. 3. (Color online) Two-photon temporal coincidences exhibit damped and overdamped oscillations with the space period $d=$ $2 \mu \mathrm{m}$. Other parameters are the same as in Ref. [16].

$A(\tau)$, whose profile is governed by $H_{0}(x)$ and is manifested by an exponential decay. In Fig. 3, we have provided two typical simulations of the coincidences using the parameters in Ref. [16]. We notice that the damped oscillations shown in Fig. 3 do not obey Rabi flopping as previously reported in Refs. [14-16,18,30]. The origin of this difference comes from the periodic modulation of the control fields, which, in turn, modifies the joint-detection patterns. The minimum coincidences appear at the zero solutions of $H_{0}(x)$. The lower curve in Fig. 3 gives the overdamped case in which even a single oscillation is not fully observable because of the fast exponential decay. Another noticeable feature is that the joint spectrum is broadened in a single oscillation due to the diffraction interference.

Next, we look at the two-photon temporal correlation mainly characterized by the phase-matching condition. That is, the natural spectral width is much narrower than the resonance linewidth such that the intrinsic mechanism of biphoton generation is partially or even fully washed out. (The latter case requires much higher optical depth.) In such a case, Eq. (9) becomes

$B(\tau)=\int_{-d / 2}^{d / 2} d x \cos \left(\frac{\pi x}{d}\right) \int d \omega \operatorname{sinc}\left(\frac{\Delta k L}{2}\right) e^{i(\Delta k L / 2-\omega \tau)}$,

which can be numerically evaluated. In Fig. 4, we have plotted the coincidence counting rate with the space period $d=2 \mu \mathrm{m}$ plus taking the third-order nonlinearity [Eq. (9)] into account. As illustrated in Fig. 4(a), most of the features appearing in previous studies [for instance, see Fig. 4(b)] can be observed. For example, the sharp peak in the leading edge of the two-photon coincidence counts represents the Sommerfeld-Brillouin precursor at the biphoton level, as reported in Ref. [31]. One difference from previous results in the literature $[15,16,21]$ is that, at the tail in Fig. 4(a), several small bumps emerge instead of a smooth exponential decay. Another difference is that the coherence time is extended. In Fig. 4(a), the coherence time is extended by more than $1 \mu \mathrm{s}$. However, without the induced
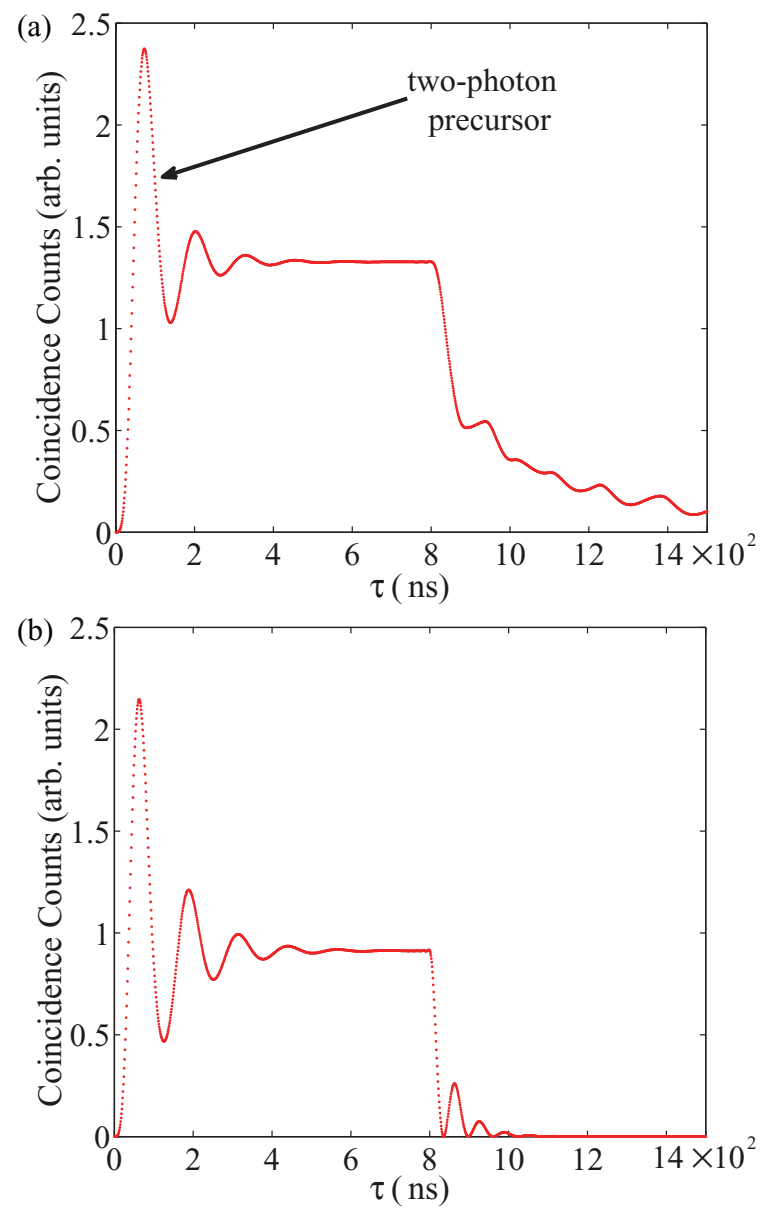

FIG. 4. (Color online) Two-photon temporal coincidences: (a) modulated by an EIG with the space period $d=2 \mu \mathrm{m}$. Other parameters are chosen as $L / v_{0}=800 \mathrm{~ns}, \Omega_{c}=5 \gamma_{31}, \gamma_{31}=2 \pi \times$ $3 \mathrm{MHz}$, and $\gamma_{21}=0.6 \gamma_{31}$. (b) Without the induced grating. Same parameters are chosen as in (a).

grating as shown in Fig. 4(b), the coherence time is only about $800 \mathrm{~ns}$. Alternatively, the joint spectrum of the biphotons is narrowed. This spectrum narrowing is a result of the spatial modulation of the control fields plus the modulated group velocities of the anti-Stokes field. Without the use of the cavity, the spectrum narrowing achieved here is useful for producing narrow-band biphotons with higher spectral brightness. If the optical depth of the medium could be made high enough, the two-photon temporal correlation would be closer to a square-wave pattern as usually observed in the SPDC process. Those small bumps would become discrete step functions at the tail, which can be verified from Eq. (14). Since this looks more like an ideal case and might not be detectable in the experiment, we will not offer further discussions here.

Before ending the discussions, in Secs. II A and II B, we have analyzed how to shape the entangled Stokes-anti-Stokes temporal wave form with the use of EIG. The extension of the idea to be used on a nonlinear crystal would be interesting. Although it is easy to design a diffraction grating within or at the output surface of the crystal, it is difficult to modulate the dispersion periodically and spatially. Therefore, it is very challenging to fully recover the features obtained here in nonlinear crystals. 


\section{SUMMARY}

To summarize, here, we have proposed a method to engineer the two-photon temporal wave packets by utilizing an EIG. The method distinguishes itself from previous research by the appearance of several features. First, the induced grating influences both the linear and the nonlinear susceptibilities. As a consequence, this will shape the biphoton wave packets through both the dispersive properties of the medium and the periodic nonlinear optical responses. Second, the induced (hybrid) nonmaterial grating directs the output anti-Stokes field into different angular positions, especially into its zerothorder diffraction. Third, the modulated biphoton wave packets exhibit different profiles compared with previous studies. For example, the damped oscillations do not coincide with the Rabi oscillations as observed in Refs. [14-16]. The decayed square-wave pattern shows small bumps at the tail, which, to the best of our knowledge, have never been discovered in the literature. Fourth, the spectral brightness and emission angle can further be engineered by the induced grating. This paper is important not only because it explores another application of the EIG, but also because the shaped biphoton wave packets hold applications in certain protocols of quantum information, quantum communications, and quantum cryptography. For instance, the properties ascribed before can be used to direct the propagation of single photons and to improve the efficiency of detecting photons in free space due to the diffraction. The broadened or narrowed bandwidth could be useful for coherent absorption and reemission of photons based on the interface between atoms and photons. The effect of EIG on the transverse correlation of entangled photons may be interesting and worth studying. However, such an issue is beyond the scope of the current paper and might be addressed somewhere else.

\section{ACKNOWLEDGMENTS}

We gratefully acknowledge insightful discussions with M. H. Rubin, K.-H. Luo, and Xiaoshun Jiang. J.W. and M.X. were supported, in part, by the National Science Foundation (USA). J.W. also acknowledges financial support by 111 Project No. B07026 (China). S.D. was supported by the Hong Kong Research Grants Council (Project No. HKUST600809).
[1] K. Hammerer, A. S. Sørensen, and E. Polzik, Rev. Mod. Phys. 82, 1041 (2010).

[2] V. Giovannetti, S. Lloyd, and L. Maccone, Phys. Rev. Lett. 96, 010401 (2006).

[3] A. Valencia, G. Scarcelli, and Y.-H. Shih, Appl. Phys. Lett. 85, 2655 (2004).

[4] Y.-H. Shih, Rep. Prog. Phys. 66, 1009 (2003).

[5] A. Pe'er, B. Dayan, A. A. Friesem, and Y. Silberberg, Phys. Rev. Lett. 94, 073601 (2005).

[6] M. Bellini, F. Marin, S. Viciani, A. Zavatta, and F. T. Arecchi, Phys. Rev. Lett. 90, 043602 (2003).

[7] M. Hendrych, X. Shi, A. Valencia, and J. P. Torres, Phys. Rev. A 79, 023817 (2009).

[8] A. Valencia, A. Ceré, X. Shi, G. Molina-Terriza, and J. P. Torres, Phys. Rev. Lett. 99, 243601 (2007).

[9] S. E. Harris, Phys. Rev. A 78, 021807(R) (2008).

[10] M. B. Nasr, S. Carrasco, B. E. A. Saleh, A. V. Sergienko, M. C. Teich, J. P. Torres, L. Torner, D. S. Hum, and M. M. Fejer, Phys. Rev. Lett. 100, 183601 (2008).

[11] A. B. U'Ren, R. K. Erdmann, M. de la Cruz-Gutierrez, and I. A. Walmsley, Phys. Rev. Lett. 97, 223602 (2006).

[12] O. Kuzucu, M. Fiorentino, M. A. Albota, F. N. C. Wong, and F. X. Kärtner, Phys. Rev. Lett. 94, 083601 (2005).

[13] T. E. Keller and M. H. Rubin, Phys. Rev. A 56, 1534 (1997).

[14] V. Balić, D. A. Braje, P. Kolchin, G. Y. Yin, and S. E. Harris, Phys. Rev. Lett. 94, 183601 (2005).

[15] S. Du, P. Kolchin, C. Belthangady, G. Y. Yin, and S. E. Harris, Phys. Rev. Lett. 100, 183603 (2008).
[16] S. Du, J.-M. Wen, and M. H. Rubin, J. Opt. Soc. Am. B 25, C98 (2008).

[17] J.-M. Wen and M. H. Rubin, Phys. Rev. A 74, 023808 (2006); 74, 023809 (2006).

[18] J.-M. Wen, S. Du, and M. H. Rubin, Phys. Rev. A 75, 033809 (2007); S. Du, J.-M. Wen, M. H. Rubin, and G. Y. Yin, Phys. Rev. Lett. 98, 053601 (2007).

[19] S. Sensarn, G. Y. Yin, and S. E. Harris Phys. Rev. Lett. 103, 163601 (2009).

[20] J. F. Chen, S. Zhang, H. Yan, M. M. T. Loy, G. K. L. Wong, and S. Du, Phys. Rev. Lett. 104, 183604 (2010).

[21] S. Du, J.-M. Wen, and C. Belthangady, Phys. Rev. A 79, 043811 (2009).

[22] B. L. Lü, W. H. Burkett, and M. Xiao, Opt. Lett. 23, 804 (1998).

[23] H. Y. Ling, Y.-Q. Li, and M. Xiao, Phys. Rev. A 57, 1338 (1998).

[24] L. E. E. de Araujo, Opt. Lett. 35, 977 (2010).

[25] M. Mitsunaga and N. Imoto, Phys. Rev. A 59, 4773 (1999).

[26] G. C. Cardoso and J. W. R. Tabosa, Phys. Rev. A 65, 033803 (2002).

[27] A. W. Brown and M. Xiao, Opt. Lett. 30, 699 (2005).

[28] See, for example, S. E. Harris, Phys. Today 50, 36 (1997); M. Xiao, Y.-q. Li, S. Z. Jin, and J. Gea-Banacloche, Phys. Rev. Lett. 74, 666 (1995).

[29] M. H. Rubin, D. N. Klyshko, Y.-H. Shih, and A. V. Sergienko, Phys. Rev. A 50, 5122 (1994).

[30] J.-M. Wen, S. Du, Y. P. Zhang, M. Xiao, and M. H. Rubin, Phys. Rev. A 77, 033816 (2008).

[31] S. Du, C. Belthangady, P. Kolchin, G. Y. Yin, and S. E. Harris, Opt. Lett. 33, 2149 (2007). 